\title{
Arbeitslosigkeit, soziale Netzwerke und gesundheitliche Ungleichheiten
}

\author{
Gerhard Krug, Stefan Brandt, Markus Gamper, \\ André Knabe und Andreas Klärner
}

\section{Überblick}

- Arbeitslosigkeit führt zu Beeinträchtigungen der physischen und psychischen Gesundheit.

- Es existieren zwei wichtige Thesen zur Rolle sozialer Netzwerke für diesen Zusammenhang.

- These 1: Arbeitslosigkeit verändert soziale Netzwerke so, dass sie ihre positive Funktion für die Gesundheit nicht mehr erfüllen (Mediatorthese).

G. Krug $(\bowtie)$

Nürnberg, Deutschland

E-Mail: gerhard.krug@iab.de

S. Brandt

Berlin, Deutschland

E-Mail: brandt@tu-berlin.de

M. Gamper

Köln, Deutschland

E-Mail: m.gamper@uni-koeln.de

A. Knabe

Rostock, Deutschland

E-Mail: andre.knabe@uni-rostock.de

A. Klärner

Braunschweig, Deutschland

E-Mail: andreas.klaerner@thuenen.de 
- These 2: Arbeitslosigkeit lässt soziale Netzwerke unverändert und Personen mit ressourcenreichen Netzwerken leiden weniger unter Gesundheitseinbußen durch Arbeitslosigkeit (Moderatorthese).

- Zu beiden Thesen existiert bisher nur wenig empirische Evidenz.

- Die wenigen quantitativen Analysen messen Netzwerke nur indirekt und sind durch fehlende Längsschnittdaten in ihrer Aussagekraft stark eingeschränkt.

- Qualitative Studien würden davon profitieren, Netzwerkeinflüsse stärker zu systematisieren und sie dadurch quantitativen Analysen zum Test zugänglich zu machen.

\section{$1 \quad$ Einleitung}

Der Verlust der Erwerbstätigkeit stellt für viele Betroffene ein Ereignis dar, das in ihren Lebenslauf eingreift, dadurch Stress auslöst (Pearlin 1989) und infolgedessen die Gesundheit negativ beeinflussen kann. Metaanalysen zeigen, dass Arbeitslose einen schlechteren Gesundheitszustand und ein um wenigstens 1,6fach höheres Mortalitätsrisiko aufweisen als beschäftigte Personen (Herbig et al. 2013; Norström et al. 2014; Roelfs et al. 2011). Dabei zeigt sich, dass Arbeitslosigkeit vor allem mit einer geringeren psychischen, aber auch physischen Gesundheit sowie teilweise mit einem riskanteren Gesundheitsverhalten (insbesondere bezüglich des Tabakkonsums) einhergeht (Freyer-Adam et al. 2011; Khlat et al. 2004; Paul und Moser 2009; McKee-Ryan et al. 2005).

Auswertungen der Studien „Gesundheit in Deutschland aktuell“ von 2010 und 2012 (Kroll et al. 2016) und aktuelle Krankenkassendaten (Knieps und Pfaff 2016) zeigen, dass psychische Probleme (Depressionen, Angststörungen etc.) bei Arbeitslosen gehäuft auftreten, diese ihren Gesundheitszustand deutlich schlechter einschätzen sowie dass sie häufiger rauchen und seltener Sport treiben als Erwerbstätige. Während internationale Studien eher auf einen höheren Alkoholkonsum bei Arbeitslosen hinweisen (Henkel 2011), gibt es diesbezüglich für Deutschland keine nennenswerten Unterschiede: Arbeitslose Frauen konsumieren sogar seltener Alkohol in gesundheitlich bedenklichem Ausmaß (vgl. dazu auch Eggs et al. 2014).

Arbeitslosigkeit beeinflusst auch den Konsum von angemessenen und gesunden Lebensmitteln wie frisches Obst und Gemüse und einer warmen Mahlzeit am Tag negativ. In der internationalen Forschung wird dies als 
„food insecurity“ bezeichnet. In den USA wurden etwa in einer Studie zu den Auswirkungen der Rezession von 2007-2009 gezeigt, dass Arbeitslosigkeit und „food insecurity“ stark korrelieren (Huang et al. 2016). Auswertungen des sozio-ökonomischen Panels (SOEP) zeigen, dass der Anteil der Ausgaben für Lebensmittel am Haushaltsbudget mit 19,2 \% bei Arbeitslosen deutlich höher, die absolute Summe von 205 EUR aber im Vergleich zu Erwerbstätigenhaushalten (13,7\% und 362 EUR) erheblich geringer ist (Pfeiffer et al. 2016).

Eine Reihe theoretischer Analysen geht davon aus, dass soziale Netzwerke für die gesundheitlichen Auswirkungen der Arbeitslosigkeit eine wichtige Rolle spielen. Dabei geht ein Teil der Literatur davon aus, dass Arbeitslosigkeit Auswirkungen auf das Netzwerk hat und diese Veränderung im Netzwerk sich wiederum auf die Gesundheit auswirkt (Mediatoreffekt, siehe z. B. Jahoda 1981; Warr 1987). Ein anderer Teil nimmt an, dass Netzwerke die negativen Folgen des durch Arbeitslosigkeit entstehenden Stresses auf die Gesundheit verringern (Moderatoreffekt, siehe z. B. Cassel 1976; Cobb 1976; Atkinson et al. 1986).

Der vorliegende Beitrag liefert einen Überblick über empirische Analysen zum Thema Netzwerke und Arbeitslosigkeit. Im Allgemeinen wird sowohl in der Alltagssprache als auch in der Wissenschaft mit dem Begriff Arbeitslosigkeit das Fehlen von Erwerbsarbeit bezeichnet (vgl. zum Folgenden auch Ludwig-Mayerhofer 2018). Damit können auch Personen, die einer aus individueller und gesellschaftlicher Sicht produktiven Arbeit nachgehen (z. B. im Haushalt oder im Ehrenamt) in diesem Sinne arbeitslos sein. In Deutschland werden sogar gemäß der sozialrechtlichen Definition der Bundesagentur für Arbeit Personen als arbeitslos in der amtlichen Statistik geführt, die einer Erwerbstätigkeit nachgehen, dies aber im Umfang von weniger als 15 Wochenstunden tun.

Eine Alternative wäre der Begriff Erwerbslosigkeit, der einerseits genau auf das Fehlen einer entlohnten Tätigkeit rekurriert. Andererseits wird der Begriff aber auch vom Statistischen Bundesamt verwendet, um damit ein alternatives, international vergleichbares Messkonzept zu bezeichnen. Dieses orientiert sich am Labour-ForceKonzept der International Labour Organization (ILO) und bezeichnet Personen als erwerbslos, die weniger als eine Stunde pro Woche einer Erwerbsarbeit nachgehen.

Im Folgenden wird - auch im Anschluss an die von uns referierte Literatur meist von Arbeitslosigkeit gesprochen, wobei der Begriff Erwerbslosigkeit damit weitgehend synonym verwendet wird. Damit ist explizit keine bewertende Aussage zu nicht entlohnten Arbeiten verbunden.

Zunächst wird in Abschn. 2 der Forschungsstand zum Thema Arbeitslosigkeit und Gesundheit referiert. In Abschn. 3 wird dann die konkrete Bedeutung sozialer Netzwerke in Bezug auf Arbeitslosigkeit und Gesundheit thematisiert. In Abschn. 3.1 werden empirische Forschungsergebnisse zur Rolle der Netzwerke 
als Mediator und in Abschn. 3.2 die Forschung zur Rolle als Moderator zusammenfasst. Abschn. 4 schließt mit einer Zusammenfassung und Bewertung des Forschungsstandes.

\section{Arbeitslosigkeit und Gesundheit}

Der negative Zusammenhang zwischen Arbeitslosigkeit und Gesundheit wurde vielfach belegt. Gewöhnlich wird der These der Verursachung gesundheitlicher Probleme durch Arbeitslosigkeit die These der Selektion von Personen mit schlechterer Gesundheit entgegengesetzt. Der Großteil der Literatur geht allerdings von der Verursachungsthese aus (Kroll et al. 2016; Brand 2015; Wanberg 2012).

In ihrer Metaanalyse berichten Paul und Moser (2009), dass sich die meisten Forschungsarbeiten zum Thema Arbeitslosigkeit und Gesundheit mit den Auswirkungen auf mentale oder allgemeine Gesundheit befassen. Physische Gesundheit spielt hier eher eine untergeordnete Rolle. Dies gilt auch noch, wenn man sich auf Analysen mit explizit kausalanalytischem Design beschränkt.

Cygan-Rehm et al. (2017) berichten negative Effekte auf die mentale Gesundheit bei den Befragten in den USA, Großbritannien, Australien und Deutschland. Die Ergebnisse von Mandemakers und Monden (2013) zeigen zudem, dass der negative Effekt auf die mentale Gesundheit vom Bildungsniveau abhängt. Dabei leiden höher gebildete Arbeitslose weniger unter Gesundheitseinbußen, da ihre Aussichten auf Wiederbeschäftigung besser sind. Zwar berichten Mandemakers und Monden (2013) von einem negativen Gesundheitstrend vor der Arbeitslosigkeit, interpretieren diese aber nicht als Beleg für Gesundheitsselektion, sondern als negative Folgen der Antizipation von Arbeitslosigkeit. Auch Young (2012) kann negative Auswirkungen auf die mentale Gesundheit für die USA aufzeigen. Diese lassen sich weder durch den arbeitslosigkeitsbedingten Einkommensverlust noch durch die (nicht) Verfügbarkeit einer Krankenversicherung erklären. Zudem kehrt der Gesundheitszustand, gemessen anhand einer Depressionsskala, auch nach erneuter Erwerbsaufnahme nicht auf das Niveau vor der Arbeitslosigkeit zurück.

Für Italien können Minelli et al. (2014) dagegen keine kausale Arbeitslosigkeitseffekte finden. Sie beziehen sich jedoch auf die selbsteingeschätzte Gesundheit. Bezogen auf Finnland finden Böckerman und Ilmakunnas (2009) ebenfalls keine kausalen Auswirkungen von Arbeitslosigkeit auf die selbsteingeschätzte Gesundheit, sie zeigen aber, dass Personen, die irgendwann arbeitslos werden, bereits vorher einen schlechteren Gesundheitszustand aufwiesen. Sie 
interpretieren dies als Beleg für die These der direkten Selektion. Tøge und Blekesaune (2015) berichten hingegen einen starken negativen kausalen Effekt der Arbeitslosigkeit auf die selbsteingeschätzte Gesundheit in 28 Staaten der EU, der mit der Dauer der Arbeitslosigkeit zunimmt. Da sie jedoch keine gleichzeitigen negativen Gesundheitstrends vor Eintritt in Arbeitslosigkeit finden, schließen sie die Selektionsthese aus. Nach Pearlman (2015) beeinflusst Arbeitslosigkeit in Folge von Firmenschließung selbsteingeschätzte Gesundheit negativ, während Arbeitslosigkeit aus anderen Gründen die Gesundheit nicht beeinflusst. Gebel und Voßemer (2014) finden zwar statistisch negative Auswirkungen auf die Lebenszufriedenheit, aber keine Effekte auf die Gesundheitszufriedenheit der Befragten in Deutschland. Sie interpretieren ihre Ergebnisse als Beleg für negative Kausaleffekte auf die psychische und nicht auf die physische Gesundheit. Dem entgegenstehend findet Schmitz (2011) zunächst negative gesundheitliche Folgen der Arbeitslosigkeit auf Gesundheitszufriedenheit, mentale Gesundheit und Krankenhausaufenthalte, aber nicht für Arbeitslose, die ihre Arbeit aufgrund einer Betriebsschließung verloren haben. Da diese Gruppe von Arbeitslosen im Gegensatz zu den anderen Arbeitslosen keine gesundheitlichen Auswirkungen zeigt, deutet dies für den Autor auf reine Selektion krank gewordener Beschäftigter in die Arbeitslosigkeit hin und nicht auf negative Gesundheitseffekte der Arbeitslosigkeit selbst. Zu ähnlichen Ergebnisse kommt auch Salm (2009) für die USA. Er findet für durch Betriebsschließungen arbeitslos gewordene Arbeitnehmer keine Auswirkungen auf die physische oder mentale Gesundheit und schließt daraus gegen das Vorliegen kausaler Effekte der Arbeitslosigkeit.

Auf Basis von norwegischen Daten können Black et al. (2012) physische Gesundheit, hier Koronarerkrankungen, herausarbeiten. Korpi (2001) analysiert schwedische Daten und berichtet unter Verwendung kausalanalytischer Verfahren zwar keine Auswirkungen der aktuellen Arbeitslosigkeit auf die Gesundheit, wohl aber negative Auswirkungen der vergangenen Arbeitslosigkeitsdauer. In einer weiteren Analyse, die jedoch keine Kontrolle unbeobachteter Drittvariablen ermöglicht, kann der Autor einen Zusammenhang zwischen schlechter Gesundheit und dem erhöhten Risiko, arbeitslos zu werden, finden. Damit bestätigt er nach eigener Aussage sowohl die Kausations- als auch die Selektionsthese.

Weitere Arbeiten befassen sich mit Gesundheitsverhalten, welches sich letztendlich auf die physische Gesundheit auswirken kann. Marcus (2014) findet signifikante positive Auswirkungen der Arbeitslosigkeit auf den Zigarettenkonsum und den Body-Mass-Index in Deutschland. Dem widersprechen jedoch die Analysen von Schunck und Rogge (2012), die ebenfalls die Auswirkungen der 
Arbeitslosigkeit auf das Rauchverhalten in Deutschland untersuchen, jedoch keinen signifikanten Effekt der Arbeitslosigkeitsdauer finden.

Qualitative Forschungsarbeiten fokussieren vornehmlich auf den Umgang der Betroffenen mit der Situation, arbeitslos zu sein. Basierend auf den Ergebnissen ihrer Analyse von Gruppendiskussionen mit Langzeitarbeitslosen beschreibt Morgenroth (2002, 2003) Erwerbslosigkeit etwa als Trauerprozess aus dem depressive Zirkel resultieren können. Ausgangspunkt der Entstehung dieser Zirkel ist, dass die emotionale Besetzung von Erwerbsarbeit als verlorenem Objekt nicht aufgegeben wird. An die Stelle einer konstruktiven Trauerarbeit im Umgang mit dem Verlust des Arbeitsplatzes treten stattdessen als ,pathologische Entgleisung“ Formen der Verarbeitung, durch die diese emotionale Besetzung aufrechterhalten wird und die sich als Teufelskreise depressiver Reaktionen selbst beständig verstärken (vgl. Morgenroth 2003, S. 23).

In seiner qualitativen Untersuchung von Handlungs- und Deutungspraktiken im Kontext von und bezogen auf Erwerbslosigkeit stellt Rogge (2013) dar, wie Menschen Erwerbslosigkeit auf unterschiedliche Weise erfahren. Basierend auf einer identitätstheoretischen Heuristik beschreibt Rogge Wirkungsweisen von Erwerbslosigkeit als Zusammenspiel kontextueller und individueller Prozesse (ebd., S. 64 ff.). Diese Wirkungsweisen fasst Rogge in fünf biografischen Identitätsmodi zusammen (,Umstellung des Selbst“, „Befreiung des Selbst“, „Kampf um das Selbst“, „Verfall des Selbst“, „Transformation des Selbst“), wobei die psychische Belastung von Erwerbslosigkeit mit dem jeweiligen Modus variieren kann. Vor allem im Rahmen des „Kampf um das Selbst“ kommt es zu einer grundlegenden Erschütterung des Selbstbildes durch Inkongruenzempfinden und Wertlosigkeitsgefühle, während im Modus „Verfall des Selbst“ Arbeitslosigkeit als schädigend erfahren wird und irreversible Wirkungen hervorbringen kann. Entscheidend für den Modus, in dem Menschen Erwerbslosigkeit durchleben, sind neben Lebensformen, sowie der Ausstattung mit ökonomischen Ressourcen und kulturellem Kapital nicht zuletzt Bezugspersonen im sozialen Beziehungsnetzwerk Erwerbsloser. Mit dem Auftrennen ,in Personen, die einerseits Arbeitslosigkeit (unterstelltermaßen) stigmatisieren, und andererseits Personen, die sie (vermeintlich) entstigmatisieren, normalisieren oder alternative und solidarische Deutungen vertreten“ (ebd. S. 272), beschreibt Rogge eine normative Spaltung persönlicher Beziehungsnetzwerke. Aus dieser Spaltung resultiert nicht nur eine selektive Hinwendung zu bzw. Abwendung von Beziehungspartnerinnen und -partnern, sondern Rogge schätzt diese Spaltung als „höchst relevant für die psychische Gesundheit von Arbeitslosen“ (ebd.) ein. 


\section{$3 \quad$ Die Rolle von Netzwerken für den Zusammenhang zwischen Arbeitslosigkeit und Gesundheit}

Der Großteil der Literatur geht davon aus, dass Arbeitslosigkeit die Gesundheit negativ beeinflusst. Netzwerken können in diesem Zusammenhang zwei unterschiedliche Rollen zukommen, die in der Literatur oft nicht deutlich voneinander getrennt werden. Zum einen können Netzwerke einen der kausalen Pfade darstellen, über den sich Arbeitslosigkeit negativ auf die Gesundheit auswirkt. Jahoda (1981) führt etwa eine Reihe von Funktionen von Erwerbsarbeit an, deren Verlust zu gesundheitlichen Einbußen führt. Sie benennt das Einkommen als die manifeste Funktion von Arbeit und eine Reihe von latenten Funktionen: die Strukturierung des Tagesablaufes, die Teilhabe an kollektiven Zielen, Aktivität, Status und Identität und schließlich soziale Kontakte. In diesem Fall würden Netzwerke dazu beitragen die Gesundheitsauswirkungen von Arbeitslosigkeit hervorzubringen. Man spricht auch davon, dass Netzwerke den Zusammenhang von Arbeitslosigkeit und schlechtem Gesundheitszustand vermitteln und damit einen Mediator darstellen (siehe Abb. 1). Werden die Netzwerke geschwächt, so können Sie ihre gesundheitsförderliche Funktion nicht mehr erfüllen und damit kommt es zu einer schlechteren Gesundheit der Arbeitslosen.

Zum andern können Netzwerke auch helfen, die negativen Effekte der Arbeitslosigkeit abzumildern. Diese These der Abschwächung negativer Auswirkungen geht im Gegensatz zur Hervorbringungsthese davon aus, dass die Netzwerke grundsätzlich unverändert bleiben. Sie besagt, dass Arbeitslose mit einem

Mediatorthese

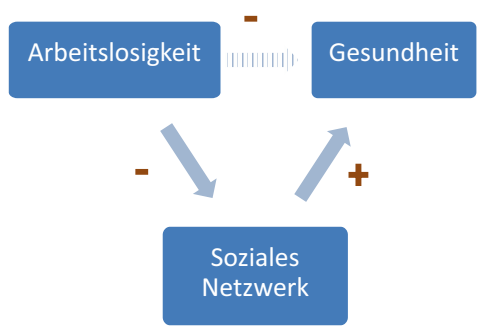

Puffer- bzw. Moderatorthese

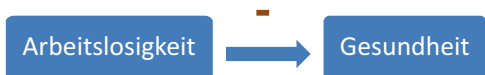

Soziales

Netzwerk

Abb. 1 Arbeitslosigkeit, Netzwerke und Gesundheit - Mediator- und Moderatorthese. (Quelle: eigene Darstellung) 
ressourcenstarken Netzwerk die negativen Auswirkungen wie Einkommens- oder Sinnverlust besser kompensieren können. Das Netzwerk liefert danach soziale, materielle, emotionale oder instrumentelle Unterstützung zur Bewältigung der Arbeitslosigkeit. Die These der Abschwächung wird auch oft als Puffer- oder Moderatorthese (siehe Abb. 1) bezeichnet (siehe auch Kap. „Sozialer Status, soziale Beziehungen und Gesundheit").

In den folgenden beiden Abschnitten wird die Literatur zu den jeweiligen Thesen dargeboten. Dabei wird deutlich, dass sich die empirische Forschung $\mathrm{zu}$ gesundheitlichen Ungleichheiten bisher selten mit dem Test beider Thesen beschäftigt hat, obwohl zumindest die These der Hervorbringung eine der theoretischen Grundlagen der Forschung zu Arbeitslosigkeit und Gesundheit darstellt.

\subsection{Mediatorthese: Netzwerkveränderung als Ursache für Gesundheitseffekte}

Die Mediatorthese geht von einer Kausalkette aus, bei der sich Arbeitslosigkeit im ersten Schritt negativ auf den Bestand und die Zusammensetzung des Netzwerkes auswirkt. Die veränderten Netzwerke wirken sich dann im zweiten Schritt der Kausalkette negativ auf die Gesundheit aus. Zwar nimmt die Mediatorthese eine prominente Stelle in der Erklärung negativer Gesundheitseffekte ein, indem soziale Kontakte als eine der latenten Funktionen der Arbeit beschrieben werden, die durch Arbeitslosigkeit beeinträchtigt werden. Dennoch lassen sich nur sehr wenige Arbeiten finden, welche die These einem direkten empirischen Test unterziehen.

Paul et al. (2009) berichten, dass der negative Effekt der Arbeitslosigkeit auf die mentale Gesundheit vollkommen durch Deprivation hinsichtlich der latenten Funktionen der Beschäftigung zu erklären ist. Die Autoren und Autorinnen liefern jedoch keine Analysen zur Relevanz der Deprivation im Teilbereich der „sozialen Kontakte“. Janlert und Hammarström (2009) untersuchen eine Reihe von theoretischen Konzepten zum Zusammenhang von Arbeitslosigkeit und Gesundheit, darunter auch soziale Unterstützung und Netzwerkumfang. Sie finden für keines der beiden nennenswerte Effekte. Ähnlich sind die Ergebnisse bei Creed und Macintyre (2001), sie finden unter Arbeitslosen keine Korrelation der sozialen Kontakte mit der psychischen Gesundheit, wohl aber signifikante Korrelationen der psychischen Gesundheit mit finanziellen Belastungen (manifeste Funktion) und den latenten Funktionen kollektiver Ziele, Zeitstruktur und Status. In einer 
Studie von Krug und Eberl (2018) wurden Paneldatenanalysen unter Berücksichtigung unbeobachteter Heterogenität und potenziell umgekehrter Kausalrichtungen durchgeführt. Es ergaben sich weder für die Zahl der engen Freunde (starke Kontakte) noch für die Mitgliedschaft in mindestens einem Verein (schwache Kontakte) Hinweise auf Mediationseffekte, ebenso wenig für das Haushaltseinkommen. Stattdessen wurde ein Teil der negativen Gesundheitseffekte über den geringeren subjektiven sozialen Status der Arbeitslosen vermittelt.

Während nur wenige Arbeiten sich mit der in der Mediatorthese angenommenen vollständigen Kausalkette zwischen Arbeitslosigkeit, Netzwerken und Gesundheit befassen, existieren weitaus mehr Arbeiten, welche zumindest einen Teil dieses Prozesses untersuchen. Neben dem Zusammenhang zwischen Netzwerken und Gesundheit ist dies der Einfluss von Arbeitslosigkeit auf soziale Netzwerke. So finden sich in der Literatur eine Reihe von Analysen der Veränderung von Größe und Struktur der Netzwerke von Arbeitslosen. Klärner und Knabe (2016) zeigen beispielsweise, dass der Übergang in die Arbeitslosigkeit mit dem Verlust der Gelegenheitsstruktur des Arbeitsplatzes zur Aufnahme und Pflege sozialer Beziehungen verbunden ist. Diewald (2007) berichtet, dass sich die Zahl der Freunde zwischen Kurzzeit- und Langzeitarbeitslosen unterscheidet. Dabei erleben Kurzzeitarbeitslose einen leichten Anstieg, während bei Langzeitarbeitslosen ein Rückgang der Freundeszahlen erfolgt. Atkinson et al. (1986) analysieren die sozialen Beziehungsnetzwerke von ca. 80 männlichen Arbeitsmarktteilnehmern. Sie berichten von keinem Einfluss der Arbeitslosigkeit auf die Netzwerkgröße, zeigen aber eine - nicht näher beschriebene - Veränderung der Zusammensetzung der Netzwerkmitglieder auf. Russel (1999) zeigt, dass die Netzwerke von Arbeitslosen zu einem hohen Anteil ebenso aus Arbeitslosen bestehen. Dies wirkt sich negativ auf die Verfügbarkeit sozialer Unterstützung und Hilfe bei der Stellenfindung aus. Lindsay (2009) kann auf Basis einer standardisierten Befragung unter Arbeitslosen in Glasgow nachweisen, dass Langzeitarbeitslose bei der Stellensuchende seltener auf ehemalige Arbeitskollegen zurückgreifen als andere Arbeitslose, aber ebenso häufig über den nahen Familien- und Freundeskreis suchen. In einer Analyse männlicher Arbeitsloser zeigt sich laut Jackson (1988) im Verlauf der Arbeitslosigkeit, dass sich das nicht-familiäre Netzwerk verkleinert, wodurch der Anteil familiärer Bindungen im Netzwerk steigt. In einer auf nur 60 Lehrern beruhenden, aber im Hinblick auf Netzwerke umfassenden Analyse berichten Röhrle und Hellmann (1989) von nur geringen Unterschieden hinsichtlich der Größe des Netzwerkes bei Lehrern mit zum Befragungszeitpunkt kürzerer, längerer oder keiner Arbeitslosigkeit. 
Ein weiterer Strang der Literatur untersucht die Auswirkungen von Arbeitslosigkeit auf das Ausmaß der sozialen Unterstützung und auf Kontakthäufigkeit mit Netzwerkmitgliedern. Laut Atkinson et al. (1986) sinkt durch Arbeitslosigkeit sowohl die familiäre Unterstützung als auch die Kontakthäufigkeit zu Bekannten und Freunden. Dabei zeigt eine getrennte Analyse nach Berufsstatus (Arbeiter vs. Angestellte), dass die Kontakthäufigkeit nur bei den Arbeitern nachlässt. Die Tatsache, dass Arbeiter stärker als Angestellte von finanziellen Einschränkungen durch Arbeitslosigkeit betroffen sind, kann laut den Autoren diesen Befund nicht vollständig erklären. Auch Gallie et al. (2001) belegen mit Daten aus mehreren Ländern, dass sich Arbeitslose insgesamt häufig mit Freunden und Bekannten treffen, dabei aber mit geringerer Wahrscheinlichkeit spezielle Unterstützungsleistungen erhalten, als Erwerbstätige. Röhrle und Hellmann (1989) berichten in ihrer bereits erwähnten Analyse bei Lehrern weder von Effekten der Arbeitslosigkeit auf die Häufigkeit noch auf die Intensität des Kontaktes. Dagegen waren arbeitslose Lehrer mit ihrem Netzwerk zufriedener und erhielten mehr soziale Unterstützung als ihre erwerbstätigen Kollegen.

Auch qualitative Analysen zeichnen das Auflösen oder das Wegfallen von Beziehungen zu Kolleginnen und Kollegen bzw. Freundinnen und Freunden aus der Arbeitswelt und die Reduktion auf den homogenen des Netzwerkes aus den engsten (meist familiären) Alteri nach (Cattell 2001; Stead et al. 2001). Als Ursache für die Verkleinerung und Homogenisierung von Netzwerken in Folge von Arbeitslosigkeit wird die gesellschaftliche Stigmatisierung und Abwertung von Arbeitslosen genannt (vgl. Knabe et al. 2017; Hirseland und Lobato 2014; Stead et al. 2001).

Die bezeichneten Prozesse der Verkleinerung und Homogenisierung von Netzwerken können sich negativ auf die Gesundheit auswirken. So beschreibt etwa Cattell (2001) die gesundheitlichen Folgen dieser Strukturveränderungen im Netzwerk auf Basis von 100 qualitativen Interviews mit von Arbeitslosigkeit und Armut betroffenen Bewohnerinnen und Bewohnern Londons: Unter den Befragten befinden sich viele Personen mit psychischen Erkrankungen und sehr geringem Selbstbewusstsein, darunter viele Frauen, die sich in materieller Abhängigkeit zu ihren Partnern befinden. Verkleinerung und Homogenisierung der Netzwerke infolge von Arbeitslosigkeit befördern den Mangel an sozialer Unterstützung, soziale Abhängigkeit und sozialen Druck. Gesundheitliche Probleme werden in Anbetracht dieser Dynamiken eher verschärft als abgemildert. Insbesondere die fehlende Einbindung in Erwerbsarbeit führt zu einem negativen Wohlbefinden aufgrund fehlender moralischer Unterstützung und fehlender sozialer Gelegenheiten zum Aufbau von Selbstvertrauen. 
Allerdings sind Netzwerke nicht nur Ursache von Problemen, sie können auch unterstützend bei der Bewältigung der negativen und gesundheitlich belastenden psychischen Folgen von Arbeitslosigkeit wirken. Den negativen Folgen der Arbeitslosigkeit auf ihre sozialen Netzwerke können von den Akteuren selbst Handlungsstrategien entgegengebracht werden, die auf eine Erweiterung von Handlungsspielräumen durch die Verlagerung der sozialen Aktivitäten auf soziale Kreise jenseits des Arbeitsmarktes zielen, z. B. in Nachbarschaften oder politischen Gruppen (vgl. Marquardsen 2012). Die Verfügbarkeit von Gelegenheitsstrukturen für derartige Handlungsstrategien jenseits des Arbeitsmarktes sowie die soziale und institutionelle Anerkennung alternativer sozialer Rollen zu der des Erwerbstätigen, erhöht die individuellen Handlungsmöglichkeiten zur Aneignung bzw. Verteidigung einer anerkennungswürdigen Identität, indem die eigene Persönlichkeit so inszeniert wird, dass die Arbeitslosigkeit in den Hintergrund rückt und stattdessen positiv besetzte soziale Rollen im Netzwerk betont werden (Knabe et al. 2017).

\subsection{Moderatorthese: Netzwerke als Schutz vor negativen Gesundheitseffekten der Arbeitslosigkeit}

Die These der Pufferfunktion beruht auf der Annahme, dass durch Netzwerke vermittelte soziale Unterstützung die negativen materiellen und emotionalen Folgen kritischer Lebensereignisse wie Arbeitslosigkeit reduziert und somit die Widerstandsfähigkeit zur Bewältigung der Arbeitslosigkeit stärkt (Cassel 1976; Cobb 1976; zusammengefasst z. B. bei Sattler und Diewald 2010).

So hat die Homogenisierung sozialer Beziehungen nicht nur negative Auswirkungen auf die Gesundheit. Netzwerke aus überwiegend arbeitslosen Personen können einen Schutzraum vor Stigmatisierung bieten und eine wichtige Quelle emotionaler Unterstützung sein. Stead et al. (2001) weisen in diesem Zusammenhang auf Ambivalenzen gesundheitsförderlicher und gesundheitsschädigender Mechanismen in sozialen Netzwerken hin. Sie analysieren die soziale Einbindung in benachteiligte Communities in Glasgow anhand von acht Gruppendiskussionen mit insgesamt 53 Teilnehmenden. Die Netzwerke der Befragten werden als relativ homogen in Bezug auf den sozialen Status, ressourcenarm und teilweise mit starken gruppeninternen Normen gesundheitsschädlichen Verhaltens (,pro-smoking community norms") bei gleichzeitiger Abgrenzung von übergeordneten gesellschaftlichen Werten (,,isolation from wider 
social norms") charakterisiert. Arbeitslosigkeit bildet in den Netzwerken der Teilnehmenden eher die Regel als die Ausnahme. Zwar bieten die so erforschten Beziehungsstrukturen Identifikationsmöglichkeiten und praktische Hilfe und haben somit eine Pufferfunktion für negative (psychische) Folgen von Langzeitarbeitslosigkeit, gleichzeitig wurden die Befragten in ihren Netzwerken jedoch eher zum Rauchen ermuntert, als beim Aufhören unterstützt.

Mit Blick auf verfügbare Quellen sozialer Unterstützung zeigt sich, dass soziale Netzwerke eine der ersten und wichtigsten Anlaufstellen bieten, um negative Folgen der Arbeitslosigkeit abzumildern, meist durch praktische Hilfen in Notfällen (z. B. Übernahme von ärztlichen Behandlungskosten durch Angehörige) sowie emotionalen Rückhalt (Edin und Lein 1997; Hill und Kauf 2001).

Heflin et al. (2011) untersuchen die Bewältigung von Notlagen in Familien mit geringem Einkommen auf Basis von qualitativen Interviews mit 50 Frauen aus den USA und stellen fest, dass Kosten für medizinische Notfälle in Ermangelung von staatlichen Hilfen und bereits ausgeschöpften individuellen Strategien (andere, weniger wichtige Rechnungen nicht zahlen, Schulden anhäufen etc.) insbesondere durch den Rückgriff auf soziale Netzwerke bewältigt werden. So können die Notlagen manchmal durch die finanziellen Ressourcen aus dem Netzwerk abgemildert werden. Die Hilfe bleibt jedoch unsicher und häufig auch uneffektiv und wenig nachhaltig. Noch weniger sind Netzwerke geeignet, kontinuierliche Bedarfe abzudecken. Die Folge sind Gesundheitsrisiken durch Verzicht auf Medikamente und Behandlungen wie z. B. Schmerzmittel, Kontrazeptiva und Asthmamittel oder Behandlungen beim Haus- oder Zahnarzt. Hill und Kauf (2001) beschreiben sehr detaillierte Mechanismen der Inanspruchnahme sozialer Unterstützung auf Basis einer Analyse von 16 unstrukturierten qualitativen Einzelinterviews mit erwerbstätigen und erwerbslosen Müttern, die in Haushalten mit sehr geringem Einkommen (unter 500 \$) in städtischen und ländlichen Regionen von Iowa (USA) leben. Viele der befragten Frauen leihen sich nur sehr ungern Geld. Wesentlich beliebter sind hingegen informelle Arbeitsgelegenheiten wie z. B. Babysitting für Verwandte, Freunde und Nachbarn. Soziale Unterstützung erscheint in der Analyse als wichtige Ressource im Netzwerk. Insbesondere aus dem Kreis der Familie werden von fast allen interviewten Müttern kleinere Geldgeschenke, Kleidung und Spielsachen für die Kinder oder Lebensmittel bezogen. Soziale Unterstützung von Nachbarinnen und Nachbarn sowie Freundinnen und Freunden erscheint seltener materiell als alltagspraktisch, z. B. in Form von Kinderbetreuung, Transport und Weitergabe von Kinderbekleidung. Soziale Unterstützung aus dem alläglichen Beziehungsnetzwerk wird häufiger in Anspruch genommen als institutionelle Angebote. Dennoch ist diese Form der Unterstützung häufig nur in geringem Maße verfügbar, da die Netzwerkpartner 
oft ebenfalls nur über beschränkte Ressourcen verfügen. Darüber hinaus reduziert sich das Ausmaß der aus dem Netzwerk empfangenen Unterstützung dadurch, dass sie oft nicht abgerufen wird, um sich ein Gefühl der Selbstwirksamkeit zu bewahren und nicht zu abhängig von dieser eher unzuverlässigen Form der Unterstützung zu werden. Premji und Shakya (2017) zeigen auf Basis von 30 Interviews mit Migrantinnen, dass soziale Isolation einen zentralen Mechanismus gesundheitlicher Beeinträchtigung durch Arbeitslosigkeit darstellt, neben intensivierten Suchbemühungen und verstärkten Haushalts- und Erziehungsaufgaben. Zusätzlich verweisen sie darauf, dass Arbeitslose mit höherer Wahrscheinlichkeit auf Stellen mit geringer Beschäftigungsqualität wieder in den Arbeitsmarkt zurückfinden.

Wie bereits im Fall der Mediatoreffekte sozialer Netzwerke existieren auch nur wenige quantitative Studien, die sich explizit den Moderatoreffekten sozialer Netzwerke im Fall der Arbeitslosigkeit widmen.

Atkinson et al. (1986) berichten, dass der negative Einfluss von Arbeitslosigkeit auf die psychische Gesundheit durch familiäre Unterstützung abgemildert wird. Für Netzwerkgröße und Kontakthäufigkeit liefern sie keine entsprechenden Analysen. Sie betonen aber die höhere Relevanz der Analyse außerfamiliärer Netzwerke, da im Bereich der Kernfamilie die gleichzeitige Betroffenheit von Arbeitslosigkeit die Frage nach der Unterstuitzungsfunktion hinfällig macht. Gore (1978) untersucht bei 100 Männern aus zwei Firmenschließungen, wie deren Gesundheitsentwicklung mit sozialer Unterstützung zusammenhängt. Diejenigen, die laut eigener Angaben auf soziale Unterstützung zurückgreifen konnten, wiesen mit geringerer Wahrscheinlichkeit Symptome physischer Krankheiten auf als andere Arbeitslose. Axelsson und Ejlertsson (2002) vergleichen in einer Querschnittsstudie die mentale Gesundheit bei arbeitslosen und erwerbstätigen jungen Erwachsenen. Dabei hängt Arbeitslosigkeit negativ mit der Gesundheit zusammen, wobei dieser Zusammenhang durch soziale Unterstützung abgeschwächt wird. Schwarzer et al. (1994) untersuchen mehr als 200 Übersiedler von Ost- nach Westdeutschland. Sie zeigen, dass diejenigen, die auf soziale Unterstützung zurückgreifen konnten, weniger körperliche Beschwerden aufwiesen. Dabei verweisen sie aber auch auf die negative Auswirkung von Krankheit auf die Verfügbarkeit sozialer Unterstützung. Die Studie von Milner et al. (2016) ist die einzige uns bekannte, die auf Basis einer Bevölkerungsstichprobe und mithilfe kausalanalytischer Methoden den Moderatoreffekt untersucht. Im Fokus steht die Auswirkung sozialer Unterstützung auf die mentale Gesundheit. Die Autoren verwenden die Panelstudie Household, Income and Labour Dynamics in Australia (HILDA) und bestätigen in Fixed-Effects-Analysen den Moderatoreffekt. Soziale Unterstützung wird anhand eines aus zehn 
Items bestehenden Mittelwertes gemessen, der dann allerdings zur weiteren Analyse - aus nicht näher bezeichneten Gründen - in eine polytome Variable mit den Ausprägungen niedrige, mittlere und hohe soziale Unterstützung vergröbert wird. Es zeigt sich, dass der negative Effekt der Arbeitslosigkeit auf die mentale Gesundheit durch hohe soziale Unterstützung abgeschwächt wird, jedoch nicht ganz vermieden werden kann.

\section{$4 \quad$ Zusammenfassung und Bewertung des Forschungsstandes}

Für die Betroffenen kann Arbeitslosigkeit mit einer Verschlechterung der physischen, vor allem aber auch der psychischen Gesundheit einhergehen. Dabei wird debattiert, inwiefern Arbeitslosigkeit hierbei auch ursächlich für einen schlechten Gesundheitszustand verantwortlich ist. Zur Beantwortung dieser Frage sind zum einen Forschungsdesigns nötig, die für Kausalanalysen besonders gut geeignet sind. Hier lässt sich in den quantitativen Analysen der letzten Jahre ein deutlicher Fortschritt beobachten, etwa die häufigere Verwendung von Längsschnittdaten und die Anwendung statistischer Verfahren zur Kontrolle unbeobachtbarer Drittvariablen. Zum anderen braucht es allerdings auch Analysen der kausalen Mechanismen, durch die Arbeitslosigkeit gesundheitliche Beeinträchtigungen hervorbringt. Einer dieser hypothetischen Mechanismen basiert auf der Vorstellung, dass soziale Netzwerke eine hohe Relevanz für gesundheitliche Ungleichheiten haben. In dem Maße, wie sich dieser Mechanismus nicht nur theoretisch explizieren, sondern auch empirisch bestätigen lässt, dient die Forschung hierzu auch als Beitrag zur übergeordneten Diskussion um den kausalen Einfluss der Arbeitslosigkeit im Unterschied zu Selektionseffekten.

Daher erstaunt zunächst die vergleichsweise geringe Zahl empirischer Forschungsarbeiten, die sich mit der Rolle sozialer Netzwerke für den Zusammenhang zwischen Arbeitslosigkeit und Gesundheit befassen. Es verwundert umso mehr, als die Literatur zur Begründung erwarteter negativer Zusammenhänge fast routineartig Jahodas (1981) Konzept des Verlusts latenter Funktionen der Arbeit rekurriert, zu denen auch die soziale Integration gehört. Der theoretisch behauptete hohe Stellenwert sozialer Netzwerke spiegelt sich aber weder in einer entsprechenden Zahl von Forschungsarbeiten, die sich mit diesem Mediatoreffekt, noch von Forschungsarbeiten, die sich mit dem angrenzendem Thema der Moderatoreffekte von Netzwerken beschäftigen, wider.

Die Forschung zu Moderator- und Mediatoreffekten sozialer Netzwerke bleibt allerdings nicht nur mengenmäßig hinter der Forschung zu Netzwerken und 
Gesundheit einerseits und Arbeitslosigkeit und Gesundheit andererseits zurück. Im Gegensatz zur Forschung zu Arbeitslosigkeit und Gesundheit spielen explizit kausalanalytische Forschungsdesigns kaum eine Rolle. So basieren viele Analysen auf sehr kleinen und vermutlich sozial selektiven Stichproben, es wird keine erwerbstätige Vergleichsgruppe herangezogen und es werden keine Paneldaten und damit verbundene statistische Verfahren der Kausalanalyse verwendet. Sicherlich kann dies vor allem älteren Arbeiten keinesfalls zum Vorwurf gemacht werden, doch fehlt es mit Ausnahme von Milner et al. (2016) und Krug und Eberl (2018) an neuern Arbeiten, welche die aktuellen Möglichkeiten der statistischen Datenanalyse für das Thema nutzbar machen.

Im Gegensatz zur Forschung zu sozialen Netzwerken und Gesundheit fällt zudem auf, dass kaum solche MessInstrumente eingesetzt werden, die zu einer adäquaten Erfassung von Netzwerken und ihren Strukturen geeignet wären. Der Grund hierfür liegt wohl größtenteils darin, dass vor allem die Literatur zu Moderatoreffekten weniger über die tatsächlichen Netzwerke, ihre Strukturen und die darüber vermittelten Ressourcen argumentiert. Vielmehr wird auf das Konzept der „sozialen Unterstützung“, oft sogar auf die lediglich „,wahrgenommene soziale Unterstützung" Bezug genommen wird. Hinzu kommt, dass der Aufwand für die Erhebung von egozentrierten Netzwerken sehr hoch ist und daher in Umfragen kaum Anwendung findet. Hier müsste ein spezielles Netzwerkerhebungsverfahren konstruiert und getestet werden - welches auf Gesundheitsaspekte zugeschnitten ist. Hier besteht große Handlungsrelevanz national wie auch international.

Erste qualitative Studien zeigen mögliche Mechanismen der Einflussnahme sozialer Beziehungsnetzwerke auf den Zusammenhang zwischen Arbeitslosigkeit und Gesundheit auf. Eine systematische Kategorisierung von Netzwerkeinflüssen auf den Zusammenhang zwischen Arbeitslosigkeit und Gesundheit fehlt bislang. Die hier vorgeschlagene Einteilung in Mediator- und Moderatoreffekte bleibt angesichts der wenigen empirischen Studien in der Literatur noch sehr fragmentarisch und thesenartig.

\section{Leseempfehlungen}

Cattell, V. (2001). Poor people, poor places, and poor health: the mediating role of social networks and social capital. Social Science and Medicine, 52 (10), S. 1501-1516. Vielzitierter Aufsatz, in dem auf Grundlage qualitativer Untersuchungen die Dynamiken von Armut und Exklusion, des Wohnumfeldes sowie von Gesundheit und Wohlbefinden unter Berücksichtigung der Rolle sozialer Netzwerk und sozialen Kapitals erörtert werden. 
Jahoda, M. (1981). Work, employment, and unemployment. Values, theories, and approaches in social research. American Psychologist, 36(2), S. 184-191. Der Aufsatz bietet eine grundlegende Übersicht zu sozialpsychologischen Theorien zu (Erwerbs-)Arbeit und Arbeitslosigkeit und betont insbesondere die latenten Funktionen der Erwerbsarbeit.

Krug, Gerhard; Eberl, Andreas (2018): What explains the negative effect of unemployment on health? An analysis accounting for reverse causality. Research in Social Stratification and Mobility, 55, S. 25-39. Eine der wenigen Arbeiten zum Mediatoreffekt, deren Analyse sich auf Paneldatenregressionen stützt. Netzwerke werden allerdings nur als einer von mehreren Mediatoren betrachtet. Als Netzwerkeigenschaften wird zudem lediglich der Umfang starker und schwacher Kontakte betrachtet.

Milner, A., Krnjacki, L., Butterworth, P., \& LaMontagne, A. D. (2016). The role of social support in protecting mental health when employed and unemployed: A longitudinal fixed-effects analysis using 12 annual waves of the HILDA cohort. Social Science and Medicine, 153, S. 20-26. Eine der wenigen Arbeiten zum Moderatoreffekt unter Verwendung von Paneldatenregressionen, der Fokus liegt aber auf sozialer Unterstützung und nicht auf netzwerktheoretischen Überlegungen.

\section{Datensätze}

- Panel Arbeitsmarkt und soziale Sicherung (PASS): jährliche Längsschnittbefragung des Instituts für Arbeitsmarkt- und Berufsforschung (IAB), seit 2007, mit Fokus auf die soziale und ökonomische Situation Arbeitsloser und Erwerbstätiger; regelmäßig Befragungsschwerpunkte zu sozialen Netzwerken sowie Gesundheit. Weitere Informationen: http://www.iab.de/de/befragungen/iab-haushaltspanel-pass.aspx

- Sozio-oekonomisches Panel (SOEP): jährliche Längsschnittbefragung des Deutschen Instituts für Wirtschaftsforschung (DIW), seit 1984 für Westdeutschland und 1990 für Ostdeutschland, mit Fokus auf die soziale und ökonomische Situation Arbeitsloser und Erwerbstätiger, regelmäßig Befragungsschwerpunkte zu sozialen Netzwerken sowie Gesundheit. Weitere Informationen: http://www.diw.de/de/soep

- Panel Armutsdynamik und Arbeitsmarkt: bundesweite qualitative Langezeituntersuchung zu Veränderungen der Lebenswege und Lebensumstände von Bezieher*innen der Grundsicherung für Arbeitssuchende 
im Zusammenhang mit Aktivierungsstrategien. Narrative Interviews u. a. zu den Einflüssen des Hilfesystems auf Lebenszusammenhänge in Mehrpersonen-Bedarfsgemeinschaften und auf Überwindung oder Verfestigung von Integrationsproblemen von Leistungsbeziehern mit Migrationshintergrund. Weitere Informationen: http://www.iab.de/138/ section.aspx/Projektdetails/k140110309

\section{Literatur}

Atkinson, T. H., Liem, J. H., \& Liem, R. (1986). The social costs of unemployment: Implications for social support. Journal of Health and Social Behavior, 27, 317-331.

Axelsson, L., \& Ejlertsson, G. (2002). Self-reported health, self-esteem and social support among young unemployed people. A population-based study. International Journal of Social Welfare, 11(2), 111-119.

Black, S. E., Devereux, P. J., \& Salvanes, K. G. (2012). Losing heart? The effect of job displacement on health. ILR Review, 68(4), 833-861.

Böckerman, P., \& Ilmakunnas, P. (2009). Unemployment and self-assessed health: Evidence from panel data. Health Economics, 18(2), 161-179.

Brand, J. E. (2015). The far-reaching impact of job loss and unemployment. Annual Review of Sociology, 41, 359-375.

Cassel, J. (1976). The contribution of the social environment to host resistance: The Fourth Wade Hampton Frost Lecture. American Journal of Epidemiology, 104(2), 107-123.

Cattell, V. (2001). Poor people, poor places, and poor health: The mediating role of social networks and social capital. Social Science and Medicine, 52(10), 1501-1516.

Cobb, S. (1976). Social support as a moderator of life stress. Psychosomatic Medicine, 38(5), 300-314.

Creed, P. A., \& Macintyre, S. R. (2001). The relative effects of deprivation of the latent and manifest benefits of employment on the well-being of unemployed people. Journal of Occupational Health Psychology, 6(4), 324-331.

Cygan-Rehm, K., Kuehnle, D., \& Oberfichtner, M. (2017). Bounding the causal effect of unemployment on mental health: Nonparametric evidence from four countries. Health Economics, 26(12), 1844-1861.

Diewald, M. (2007). Arbeitsmarktungleichheiten und die Verfügbarkeit von Sozialkapital. Die Rolle von Gratifikationen und Belastungen. Kölner Zeitschrift für Soziologie und Sozialpsychologie, 47, 183-210.

Edin, K., \& Lein, L. (1997). Making Ends Meet. How Single Mothers Survive Welfare and Low-Wage Work. New York: Russell Sage.

Eggs, J., Trappmann, M., \& Unger, S. (2014). Grundsicherungsempfänger und Erwerbstätige im Vergleich. ALG-II-Bezieher schätzen ihre Gesundheit schlechter ein. IAB-Kurzbericht, 23.

Freyer-Adam, J., Gaertner, B., Tobschall, S., \& John, U. (2011). Health risk factors and self-rated health among job-seekers. BMC Public Health, 11, 659. 
Gallie, D., Kostova, D., \& Kuchar, P. (2001). Social consequences of unemployment. An east-west Comparison. Journal of European Social Policy, 11(1), 39-54.

Gebel, M., \& Voßemer, J. (2014). The impact of employment transitions on health in Germany. A difference-in-differences propensity score matching approach. Social Science and Medicine, 108, 128-136.

Gore, S. (1978). The effect of social support in moderating the health consequences of unemployment. Journal of Health and Social Behavior, 19(2), 157-165.

Heflin, C., London, A. S., \& Scott, E. K. (2011). Mitigating material hardship. The strategies low-income families employ to reduce the consequences of poverty. Sociological Inquiry, 81(2), 223-246.

Henkel, D. (2011). Unemployment and substance use: A review of the literature (19902010). Current Drug Abuse Reviews, 4(1), 4-27.

Herbig, B., Dragano, N., \& Angerer, P. (2013). Health in the long-term unemployed. Deutsches Ärzteblatt International, 110(23-24), 413-419.

Hill, H., \& Kauff J. (2001). Living on little. Case studies of Iowa families with very low incomes. Mathematica Policy Research. https://www.mathematica-mpr.com/our-publications-and-findings/publications/living-on-little-case-studies-of-iowa-families-withvery-low-incomes. Zugegriffen: 18. Febr. 2019.

Hirseland, A., \& Ramos Lobato, P. (2014). "Die wollen ja ein bestimmtes Bild vermitteln". Zur Neupositionierung von Hilfeempfängern im aktivierenden Sozialstaat. SWS-Rundschau, 54(2), 181-200.

Huang, J., Kim, Y., \& Birkenmaier, J. (2016). Unemployment and household food hardship in the economic recession. Public Health Nutrition, 19(3), 511-519.

Jackson, P. R. (1988). Personal networks, support mobilization and unemployment. Psychological Medicine, 18(2), 397-404.

Jahoda, M. (1981). Work, employment, and unemployment. Values, theories, and approaches in social research. American Psychologist, 36(2), 184-191.

Khlat, M., Sermet, C., \& Le Pape, A. (2004). Increased prevalence of depression, smoking, heavy drinking and use of psycho-active drugs among unemployed men in France. European Journal of Epidemiology, 19(5), 445-451.

Klärner, A., \& Knabe, A. (2016). Soziale Netzwerke als Ressource für den Umgang mit Langzeitarbeitslosigkeit. WSI-Mitteilungen, 5, 354-363.

Knabe, A., Fischer, H., \& Klärner, A. (2017). Armut als relationales Konstrukt: Die (Re-) Produktion sozialer Ungleichheiten durch Stigmatisierung und Kontrollversuche in sozialen Netzwerken. In L. Behrmann, F. Eckert, A. Gefken, \& P. A. Berger (Hrsg.), ,Doing Inequality“ - Prozesse sozialer Ungleichheit im Blick qualitativer Sozialforschung (S. 167-190). Wiesbaden: Springer VS.

Knieps, F., \& Pfaff, H. (Hrsg.). (2016). Gesundheit und Arbeit. Zahlen, Daten, Fakten; mit Gastbeiträgen aus Wissenschaft, Politik und Praxis. Berlin: Medizinisch Wissenschaftliche Verlagsgesellschaft.

Korpi, T. (2001). Accumulating disadvantage. Longitudinal analyses of unemployment and physical health in representative samples of the Swedish population. European Sociological Review, 17(3), 255-273.

Kroll, L. E., Müters, S., \& Lampert, T. (2016). Arbeitslosigkeit und ihre Auswirkungen auf die Gesundheit. Ein Überblick zum Forschungsstand und zu aktuellen Daten der Studien GEDA 2010 und GEDA 2012. Bundesgesundheitsblatt, Gesundheitsforschung, Gesundheitsschutz, 59(2), 228-237. 
Krug, G., \& Eberl, A. (2018). What explains the negative effect of unemployment on health? An analysis accounting for reverse causality. Research in Social Stratification and Mobility, 55, 25-39.

Lindsay, C. (2009). In a lonely place? Social networks, job seeking and the experience of long-term unemployment. Social Policy \& Society, 9(1), 25.

Ludwig-Mayerhofer, W. (2018). Arbeitslosigkeit. In M. Abraham \& T. Hinz (Hrsg.), Arbeitsmarktsoziologie (3. Aufl., S. 155-192). Wiesbaden: VS.

Mandemakers, J. J., \& Monden, C. W. S. (2013). Does the effect of job loss on psychological distress differ by educational level? Work, Employment \& Society, 27(1), 73-93.

Marcus, J. (2014). Does job loss make you smoke and gain weight? Economica, 81(324), $626-648$.

Marquardsen, K. (2012). Aktivierung und soziale Netzwerke. Die Dynamik sozialer Beziehungen unter dem Druck der Erwerbslosigkeit. Wiesbaden: VS.

McKee-Ryan, F., Song, Z., Wanberg, C. R., \& Kinicki, A. J. (2005). Psychological and physical well-being during unemployment: A meta-analytic study. The Journal of Applied Psychology, 90(1), 53-76.

Milner, A., Krnjacki, L., Butterworth, P., \& LaMontagne, A. D. (2016). The role of social support in protecting mental health when employed and unemployed: A longitudinal fixed-effects analysis using 12 annual waves of the HILDA cohort. Social Science and Medicine, 153, 20-26.

Minelli, L., Pigini, C., Chiavarini, M., \& Bartolucci, F. (2014). Employment status and perceived health condition: Longitudinal data from Italy. BMC Public Health, 14, 946.

Morgenroth, C. (2003). Arbeitsidentität und Arbeitslosigkeit - Ein depressiver Zirkel. Aus Politik und Zeitgeschichte, 6-7, 17-24.

Morgenroth, C. (2002). Depressive Dynamik in der Arbeitslosigkeit. In T. Darmstädter (Hrsg.), Transformation der Arbeit (S. 135-152). Frankfurt a. M.: Neue Kritik.

Norström, F., Virtanen, P., Hammarström, A., Gustafsson, P. E., \& Janlert, U. (2014). How does unemployment affect self-assessed health? A systematic review focusing on subgroup effects. BMC Public Health, 14, 1310.

Paul, K. I., \& Moser, K. (2009). Unemployment impairs mental health. Meta-analyses. Journal of Vocational Behavior, 74(3), 264-282.

Paul, K. I., Geithner, E., \& Moser, K. (2009). Latent deprivation among people who are employed, unemployed, or out of the labor force. The Journal of Psychology, 143(5), 477-491.

Pearlin, L. I. (1989). The sociological study of stress. Journal of Health and Social Behavior, 30(3), 241-256.

Pearlman, J. (2015). The consequences of job displacement for health: Moderating influences of economic conditions and educational attainment. Social Science Research, 52, 570-587.

Pfeiffer, S., Oestreicher, E., \& Ritter, T. (2016). Hidden and neglected: Food poverty in the Global North: The case of Germany. World Review of Nutrition and Dietetics, 115, 16-23.

Premji, S., \& Shakya, Y. (2017). Pathways between under/unemployment and health among racialized immigrant women in Toronto. Ethnicity \& Health, 22(1), 17-35.

Röhrle, B., \& Hellmann, I. (1989). Characteristics of social networks and social support among long-term and short-term unemployed teachers. Journal of Social and Personal Relationships, 6, 463-473.

Roelfs, D. J., Shor, E., Davidson, K. W., \& Schwartz, J. E. (2011). Losing life and livelihood: A systematic review and meta-analysis of unemployment and all-cause mortality. Social Science and Medicine, 72(6), 840-854. 
Rogge, B. (2013). Wie uns Arbeitslosigkeit unter die Haut geht. Identitätsprozess und psychische Gesundheit bei Statuswechseln. Konstanz: UVK.

Russell, H. (1999). Friends in low places. Gender, unemployment and sociability. Work, Employment and Society, 13(2), 205-224.

Salm, M. (2009). Does job loss cause ill health? Health Economics, 18(9), 1075-1089.

Sattler, S., \& Diewald, M. (2010). Wechselwirkungen zwischen Arbeitslosigkeit und dem sozialen Netzwerk. In C. Stegbauer \& R. Häußling (Hrsg.), Handbuch Netzwerkforschung (S. 701-713). Wiesbaden: VS.

Schmitz, H. (2011). Why are the unemployed in worse health? The causal effect of unemployment on health. Labour Economics, 18(1), 71-78.

Schunck, R., \& Rogge, B. G. (2012). No causal effect of unemployment on smoking? A German panel study. International Journal of Public Health, 57(6), 867-874.

Schwarzer, R., Jerusalem, M., \& Hahn, A. (1994). Unemployment, social support and health complaints. A longitudinal study of stress in East German refugees. Journal of Community \& Applied Social Psychology, 4(1), 31-45.

Stead, M., MacAskill, S., MacKintosh, A. M., Reece, J., \& Eadie, D. (2001). 'It's as if you're locked in": qualitative explanations for area effects on smoking in disadvantaged communities. Health \& Place, 7(4), 333-343.

Tøge, A. G., \& Blekesaune, M. (2015). Unemployment transitions and self-rated health in Europe: A longitudinal analysis of EU-SILC from 2008 to 2011. Social Science \& Medicine, 143, 171-178

Wanberg, C. R. (2012). The individual experience of unemployment. Annual Review of Psychology, 63, 369-396.

Warr, P. (1987). Work, unemployment, and mental health. New York: Oxford University Press. Young, C. (2012). Losing a job. The nonpecuniary cost of unemployment in the United States. Social Forces, 91(2), 609-634.

Open Access Dieses Kapitel wird unter der Creative Commons Namensnennung 4.0 International Lizenz (http://creativecommons.org/licenses/by/4.0/deed.de) veröffentlicht, welche die Nutzung, Vervielfältigung, Bearbeitung, Verbreitung und Wiedergabe in jeglichem Medium und Format erlaubt, sofern Sie den/die ursprünglichen Autor(en) und die Quelle ordnungsgemäß nennen, einen Link zur Creative Commons Lizenz beifügen und angeben, ob Änderungen vorgenommen wurden.

Die in diesem Kapitel enthaltenen Bilder und sonstiges Drittmaterial unterliegen ebenfalls der genannten Creative Commons Lizenz, sofern sich aus der Abbildungslegende nichts anderes ergibt. Sofern das betreffende Material nicht unter der genannten Creative Commons Lizenz steht und die betreffende Handlung nicht nach gesetzlichen Vorschriften erlaubt ist, ist für die oben aufgeführten Weiterverwendungen des Materials die Einwilligung des jeweiligen Rechteinhabers einzuholen.

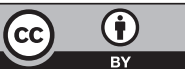

